\title{
3 Research Square

\section{Acceptability of COVID-19 Vaccination among Healthcare Workers in Sudan: A Cross Sectional Survey}

\section{Amna Khairy ( $\square$ Amnakh22@gmail.com )}

Federal Ministry of Health

\section{Esra Mahgoob}

Al-Neelain University

Mohammad Nimir

University of Warwick

Mohammed Ahmed

Sudan Medical Specialization Board

Mawahib Jubara

Federal Ministry of Health

Dalya Eltayeb

Federal Ministry of Health

Ayman Ahmed

University of Khartoum

\section{Research Article}

Keywords: COVID-19, vaccine acceptability, Healthcare workers, Sudan

Posted Date: July 26th, 2021

DOI: https://doi.org/10.21203/rs.3.rs-745232/v1

License: (c) (1) This work is licensed under a Creative Commons Attribution 4.0 International License. Read Full License 


\section{Abstract}

Introduction: Following emergency approval of COVID-19 vaccines, several studies have investigated COVID-19 vaccine acceptance and hesitancy especially among healthcare workers (HCWs). Nevertheless, the acceptability of the COVID-19 vaccine by HCWs in Sudan remains unclear. This study aims to investigate the acceptability of the COVID-19 vaccine and it's determinants among the HCWs.

Methods: A web-based cross sectional study design was used to study COVID-19 vaccine hesitancy and its associated determinants. The semi-structured questionnaire was distributed electronically, data collection took place from March-April 2021.

Results: A total of $576 \mathrm{HCWs}$ have responded to the survey with mean age of 35 years. The majority were females (53.3\%), Medical Doctors (55.4\%), and located in the capital state; Khartoum (76.0\%). The absolute refusal of COVID-19 vaccine was expressed by $16 \%$ of the respondents while $57 \%$ were willing to get vaccinated. Males were twice to four times more likely to accept the vaccine. Lower acceptability of COVID-19 vaccine was significantly associated with the nursing profession (OR $=0.35,95 \% \mathrm{Cl}: 0.15-0.82$, $P<0.00$ ), increased perceived harm from the vaccine ( $\mathrm{OR}=0.11,95 \% \mathrm{Cl}: 0.05-0.23, P<0.00)$, lack of confidence in the source of vaccine ( $\mathrm{OR}=0.16,95 \% \mathrm{Cl}: 0.08-0.31, P=0.00)$, organizations and government supervising COVID-19 vaccination process ( $\mathrm{OR}=0.31,95 \% \mathrm{Cl}: 0.17-0.58 P=0.00$ ).

Conclusion: This study highlights a moderate level of COVID-19 vaccine acceptability by the HCWs in Sudan. Effective communication of correct, regular, up-to-date evidence on the safety and effectiveness of vaccines is crucial to building trust in vaccines. Special consideration should be in place to address vaccine hesitancy among female HCWs and the nursing profession.

\section{Introduction}

The novel coronavirus that was first emerged in December 2019 and developed into a global pandemic of coronavirus disease 2019 (COVID-19) by March 2020 (1). Since then, the number of COVID-19 cases has been escalating and by 27th of June 2021, the global number of cases exceeded 179 million confirmed cases with 4 million confirmed deaths (2). The rapid transmission of the disease and the high risk to global health have pushed the governments to implement harsh restrictions and regulations to control the pandemic including social distancing and massive use of facemasks. Despite all the preventive measures, the pandemic still developing and claiming more lives worldwide particularly among the first responders, the Healthcare Workers (HCWs) (3).

First infections of COVID-19 in Sudan were detected among international travelers and returnees who arrived in the country between February and March 2020 (4). Soon later, Sudan has suffered from the widespread of infections and the local dramatic development of the pandemic in lack of adherence among the public community and noncompliance of the individuals to the prevention measures that were promoted by the Ministry of Health (5). 
The first glimpse of hope for humanity rosed up in December 2020 when the World Health Organization (WHO) has validated the use of the first COVID-19 vaccine for humans (6). By February 2021, seven vaccines were deployed globally, with priority being given to the most vulnerable groups, including mainly the HCWs (7). However, the successful deployment of all these different vaccines to reach the optimal coverage that provides community-wide protection was challenged globally by the vaccine hesitancy (8). The concept of vaccine hesitancy refers to the rejection of vaccination or delay in considering taking it despite the presence of vaccine (9). Unfortunately, vaccine hesitancy was documented even among HCWs worldwide (9-11). The vaccine hesitancy is of high risk when it spreads among the HCWs. Mainly because this group of professionals is generally perceived by the public communities as the most trusted source of information, awareness, and encouragement for vaccine intake among other professionals and the public. A recent study in Saudi Arabia showed that despite the efforts of the Ministry of Health (MoH) to spread the data on the safety and effectiveness of the COVID-19 vaccine, nearly $50 \%$ of the HCWs were reluctant to take the vaccine (12). Similarly in Congo and the United States, merely $72 \%$ and $56 \%$ of the HCWs were hesitant about taking a COVID-19 vaccine $(13,14)$. Nevertheless, a high acceptance rate among HCWs was reported in some countries, such as Greek, France, and China where the rates were 80 $\%, 76.9 \%$, and $76.4 \%$, respectively (15-17).

The Expanded Programme of Immunization (EPI) in Sudan faces a similar challenge about vaccinating the vulnerable groups, particularly HCWs, although the number of cases steadily increasing and now it, as of June 2021, exceeded 36 thousand with more nearly 3000 related deaths (2). On the 3rd of March, Sudan received the AstraZeneca vaccine through the COVAX alliance (18). There is a huge gap of information about the hesitancy and/or acceptability of the COVID-19 vaccine among the HCWs. Therefore, this study aimed to contribute to filling this gap by investigating the perception, awareness, and attitude of HCWs towards the intake of COVID-19 vaccine and determine the underpinning believes and assumptions.

\section{Materials And Methods}

\section{Study Design and Sampling:}

A cross-sectional online survey was conducted during the period of March- April 2021, the inclusion criteria were HCWs who were practicing in Sudan during the data collection period including dentists, medical laboratory technologists, nurses, physicians, Pharmacists, paramedic staff, and public health workers, who agreed to participate in the study and having access to internet connection to fill out the online questionnaire. This survey was designed and implemented by the Ministry of Health as part of the preparatory phase for launching the COVID-19 vaccination campaign in the country.

The total number of HCWs targeted for the vaccinations as estimated by the Federal Ministry of health is $400000 \mathrm{HCW}$. Assuming that $50 \%$ is the expected magnitude of vaccine acceptance, using alpha error of 0.05 and margin of error $5 \%$, the minimum required sample size was $564 \mathrm{HCWs}$. The sample size was calculated using EPI-info 7 software. Convenience sampling technique was used to recruit the 
respondents, the questionnaire was distributed through professional social media platforms that were exclusive for Sudanese HCWs.

\section{Survey Instrument:}

A semi-structured questionnaire was adopted from previous studies $(14,19)$. The questionnaire was selfadministered through a Google form and it has three major sections: (A) Background characteristics include demographic and professional information related to the knowledge and training about COVID-19 and its vaccine. (B) COVID-19 Vaccine acceptance measured with two questions: Would you take the COVID-19 vaccine when it is available (Yes, maybe, No)? and would you advise your family and friends to take the vaccine (Yes, maybe, No)? and (C) Health believes model which was used to explore the determinants for Covid-19 vaccinations acceptability. The model explored the following items: the perceived susceptibility measured by considering oneself at risk to get COVID-19 infection and the need to take the vaccine. Perceived severity was measured as the expected outcome if the subjects contracted COVID-19 infection. Perceived harm was measured by concerns related to the safety and side effects of the vaccine. The perceived benefit was measured as beliefs in the vaccine efficacy and effectiveness. Cues to action which was measured as whom subjects are intending to protect by taking the vaccine. Perceived barriers were focused on potential lack of confidence in the vaccine producers, government and organizations supervising the vaccination process. Additionally, the open-end broad question: Do you have any comment regarding COVID-19 vaccination in Sudan? was used to qualitatively explore HCWs different perspectives on vaccine acceptability and hesitancy.

\section{Data analysis:}

Data from the google sheet was cleaned and entered into IBM® SPSS ${ }^{\circledR}$ Statistics (version 25).

Descriptive analysis: This was performed in form of frequency tables was done for categorical variables. Means (M) and standard deviations (SD) were estimated for the continuous variables such as age and years of working experience. For the open-end broad question: Do you have any Comments regarding COVID-19 vaccination in Sudan? Answers were translated (if were in Arabic), coded, and common themes were identified.

Inferential analysis: Bivariate analysis and multivariable logistic regression analysis was performed to test for determinants of acceptance of COVID-19 vaccination. Only definitive Yes/No responses were included in this analysis excluding the "maybe" answers. Cross tabulation and Persons Chi-Square test was done to test associations between the outcome variables. Crude Odds Ratio (OR) was used as the estimate of the association. A $P$ value $<0.05$ was used as an indicator for the level of significance. These associations have been further adjusted using Multivariable logistic regression. Adjusted Odds Ratio (AOR), $95 \% \mathrm{Cl}$ for the AOR, and a significant level of $P<0.05$ were used.

\section{Ethical Considerations:}


The survey was conducted by the Expanded Programme of Immunization (EPI), Federal Ministry of Health as part of the country's immediate response strategy for the COVID-19 pandemic including the preparation for vaccination throughout the country. Therefore, the need for ethical approval was waived by the National COVID-19 Response Committee. However, informed consent with study explanatory statements was shared with HCWs before their voluntary enrollment in this study. Only HCWs who read and sign this informed consent and provided their responses to the questionnaire were included in this study.

\section{Results}

\section{Sociodemographic characteristics of respondents:}

A total of $576 \mathrm{HCWs}$ have participated in this study and responded to the survey. The demographic of the participants showed that females represented $53.3 \%(n=307)$ and the mean age of the participants was 35 years old $(S D=10.6)$. The majority of the participants were working in Khartoum; the capital state of Sudan 438 (76.0\%), and most of them were physicians 319 (55.4\%) (Table 1). Regarding health characteristics, around $27 \%(\mathrm{n}=155)$ were diagnosed with medical comorbidity, mainly asthma $(34.5 \%, n$ $=69)$, hypertension $(24 \%, n=48)$, and Diabetes mellites $(20.5 \%, n=41)$. Forty-five percent $(n=259)$ of the study participants have received training on COVID-19, yet only $24.5 \%(n=123)$ and $8.7 \%(n=50)$ have described their level of knowledge about COVID-19 disease and COVID-19 vaccination as "very good" or "excellent" respectively. Twenty percent $(n=115)$ had contracted COVID-19 infection before, and more than half $(52.4 \%, n=302)$ were directly or indirectly caring of COVID-19 cases (Table 1$)$. 
Table 1

Socio-Demographic and professional characteristics

\begin{tabular}{|c|c|c|}
\hline Socio-Demographic and professional characteristics & Value & $N=576$ \\
\hline Age $(M \pm S D)$ & $35.3(10.6)$ & \\
\hline \multirow[t]{2}{*}{ Gender } & Male & $\begin{array}{l}269 \\
(46.7 \%)\end{array}$ \\
\hline & Female & $\begin{array}{l}307 \\
(53.3 \%)\end{array}$ \\
\hline \multirow[t]{2}{*}{ Medical comorbidities } & Yes & $\begin{array}{l}155 \\
(26.9 \%)\end{array}$ \\
\hline & No & $\begin{array}{l}421 \\
(73.1 \%)\end{array}$ \\
\hline \multirow[t]{7}{*}{ Type of Medical Co-morbidities } & Diabetes mellites & $41(20.5 \%)$ \\
\hline & Hypertension & $48(24.0 \%)$ \\
\hline & Asthma & $69(34.5 \%)$ \\
\hline & Chronic Kidney Disease & $5(2.5 \%)$ \\
\hline & $\begin{array}{l}\text { Connective tissue } \\
\text { Disease }\end{array}$ & $5(2.5 \%)$ \\
\hline & Cancer & $6(3.0 \%)$ \\
\hline & others & $26(13.0 \%)$ \\
\hline \multirow[t]{2}{*}{ Immune Suppressing Medications } & Yes & $15(2.6 \%)$ \\
\hline & No & $\begin{array}{l}556 \\
(76.3 \%)\end{array}$ \\
\hline \multirow[t]{7}{*}{ Health Profession } & Medical Doctor & $\begin{array}{l}319 \\
(55.4 \%)\end{array}$ \\
\hline & Nurses & $17(3.0 \%)$ \\
\hline & Pharmacists & $66(11.5 \%)$ \\
\hline & $\begin{array}{l}\text { Medical laboratory } \\
\text { Scientist }\end{array}$ & $26(4.5 \%)$ \\
\hline & Public Health Workers & $86(14.9 \%)$ \\
\hline & Dentists & $26(4.5 \%)$ \\
\hline & Other(s) & $36(6.3 \%)$ \\
\hline Years of practice or working experience $(M \pm S D)$ & $10.4(9.6)$ & \\
\hline
\end{tabular}




\begin{tabular}{|c|c|c|}
\hline Socio-Demographic and professional characteristics & Value & $N=576$ \\
\hline \multirow[t]{3}{*}{ State of practice } & Khartoum & $\begin{array}{l}438 \\
(76.0 \%)\end{array}$ \\
\hline & Other than Khartoum & $\begin{array}{l}134 \\
(23.3 \%)\end{array}$ \\
\hline & I don't work in Sudan & $4(0.7 \%)$ \\
\hline \multirow[t]{2}{*}{ Any training on COVID-19 } & Yes & $\begin{array}{l}259 \\
(45.0 \%)\end{array}$ \\
\hline & No & $\begin{array}{l}317 \\
(55.0 \%)\end{array}$ \\
\hline \multirow[t]{5}{*}{ Knowledge about COVID-19 } & Excellent & $\begin{array}{l}141 \\
(24.5 \%)\end{array}$ \\
\hline & Very good & $\begin{array}{l}221 \\
(38.4 \%)\end{array}$ \\
\hline & Moderate & $\begin{array}{l}173 \\
(30.0 \%)\end{array}$ \\
\hline & Good & $29(5.0 \%)$ \\
\hline & Weak & $12(2.1 \%)$ \\
\hline \multirow[t]{5}{*}{ Knowledge about COVID-19 Vaccination } & Excellent & $50(8.7 \%)$ \\
\hline & Very good & $\begin{array}{l}123 \\
(21.4 \%)\end{array}$ \\
\hline & Moderate & $\begin{array}{l}177 \\
(30.7 \%)\end{array}$ \\
\hline & Good & $\begin{array}{l}118 \\
(20.5 \%)\end{array}$ \\
\hline & Weak & $\begin{array}{l}108 \\
(18.8 \%)\end{array}$ \\
\hline \multirow[t]{2}{*}{ Diagnosed with COVID-19 } & Yes & $\begin{array}{l}115 \\
(20.0 \%)\end{array}$ \\
\hline & No & $\begin{array}{l}461 \\
(80.0 \%)\end{array}$ \\
\hline \multirow[t]{2}{*}{$\begin{array}{l}\text { Family Members have ever been Diagnosed with COVID- } \\
19\end{array}$} & Yes & $\begin{array}{l}216 \\
(37.5 \%)\end{array}$ \\
\hline & No & $\begin{array}{l}360 \\
(62.5 \%)\end{array}$ \\
\hline $\begin{array}{l}\text { Directly or indirectly been taking care of the COVID-19 } \\
\text { patients }\end{array}$ & Yes & $\begin{array}{l}302 \\
(52.4 \%)\end{array}$ \\
\hline
\end{tabular}




\begin{tabular}{|c|c|c|}
\hline \multirow[t]{2}{*}{ Socio-Demographic and professional characteristics } & Value & $N=576$ \\
\hline & No & $\begin{array}{l}274 \\
(47.6 \%)\end{array}$ \\
\hline \multirow[t]{3}{*}{ Would you take the COVID-19 Vaccine? } & Yes & $\begin{array}{l}329 \\
(57.0 \%)\end{array}$ \\
\hline & May be & $\begin{array}{l}155 \\
(27.0 \%)\end{array}$ \\
\hline & No & $92(14.0 \%)$ \\
\hline \multirow[t]{3}{*}{ Advise friends and family to get vaccinated for COVID-19 } & Yes & $\begin{array}{l}438 \\
(60.1 \%)\end{array}$ \\
\hline & May be & $52(7.1 \%)$ \\
\hline & No & $86(11.8 \%)$ \\
\hline
\end{tabular}

\section{Vaccine acceptability, hesitancy and refusal among HCWs in Sudan}

A total of 329 (57.0\%) HCWs expressed their willingness to be vaccinated against COVID-19, while 155 (27.0\%) were hesitant, and only $92(14.0 \%)$ were refusing to take the COVID-19 vaccine (Table 1 ). Furthermore, 438 (60.1\%), 52 (7.1\%), 86 (11.8\%) have responded yes, maybe, and no, respectively, regarding advising friends and family to get vaccinated for COVID-19 (Table 1).

\section{Parameters associated with acceptability of COVID-19 vaccine}

Bivariate analysis (Table 2) showed that the mean age for those who would accept the vaccine was 36.5 years while the average age of refusers was 34.1 years, $P<0.05$. The gender of the participant was found to be strongly associated with the acceptance rate with $85.8 \%$ of men accepting the vaccine compared to $70.5 \%$ among the female participants. Vaccine acceptance significantly increased with years of working experience (Difference in Means $=2.2, P<0.05$ ), receiving training related to COVID-19 vaccination, and taking care of COVID-19 patients directly or indirectly, $p<0.05$. Those who rated their knowledge about the COVID-19 vaccination as excellent were significantly the least willing to be vaccinated $(59.4 \%), \mathrm{P}<$ 0.05 . 
Table 2

Bivariate analysis for Socio-demographic, profession, and health characteristics as determinants of COVID-19 vaccine acceptance

\begin{tabular}{|c|c|c|c|c|c|}
\hline \multirow[t]{2}{*}{ Characteristics } & \multirow[t]{2}{*}{ Value } & \multicolumn{2}{|c|}{$\begin{array}{l}\text { Vaccine } \\
\text { acceptance/refusal ( } \mathrm{N} \\
\left.=421^{1}\right)(\%)\end{array}$} & \multirow[t]{2}{*}{$\begin{array}{l}\text { Unadjusted } \\
\text { OR ( } 95 \% \\
\mathrm{Cl})\end{array}$} & \multirow[t]{2}{*}{ p } \\
\hline & & Accept (\%) & $\begin{array}{l}\text { Refuse } \\
\text { (\%) }\end{array}$ & & \\
\hline Age & Mean (sd) & $36.5(11.5)$ & $34.1(9.6)$ & & 0.04 \\
\hline \multirow[t]{2}{*}{ Gender } & Male & $181(85.8)$ & $30(14.2)$ & \multirow{2}{*}{$\begin{array}{l}2.52(1.55- \\
4.11)\end{array}$} & \multirow[t]{2}{*}{0.00} \\
\hline & Female $^{2}$ & 148(70.5) & $62(29.5)$ & & \\
\hline \multirow[t]{2}{*}{ Medical Co-morbidities } & Yes & $93(79.5)$ & $24(20.5)$ & \multirow{2}{*}{$\begin{array}{l}1.11 \\
(0.66- \\
1.88)\end{array}$} & \multirow[t]{2}{*}{0.68} \\
\hline & $\mathrm{No}^{2}$ & $236(77.6)$ & $68(22.4)$ & & \\
\hline \multirow{2}{*}{$\begin{array}{l}\text { Taking Immune suppressing } \\
\text { medications }\end{array}$} & Yes & $9(69.2)$ & $4(30.8)$ & \multirow{2}{*}{$\begin{array}{l}0.61(0 \\
.18-2.06)\end{array}$} & \multirow[t]{2}{*}{0.49} \\
\hline & $\mathrm{No}^{2}$ & $316(78.4)$ & $87(21.6)$ & & \\
\hline \multirow[t]{7}{*}{ Health Profession } & Medicine & 190(79.2) & $50(20.8)$ & $\begin{array}{l}1.03(0.93- \\
1.14)\end{array}$ & 0.5 \\
\hline & Nursing & $4(28.6)$ & 10(71.4) & $\begin{array}{l}0.35(0.15- \\
0.82)\end{array}$ & 0.00 \\
\hline & Pharmacy & $31(72.1)$ & 12(27.9) & $\begin{array}{l}0.91(0.75- \\
1.10)\end{array}$ & 0.31 \\
\hline & $\begin{array}{l}\text { Medical } \\
\text { laboratory }\end{array}$ & $13(76.5)$ & $4(23.5)$ & $\begin{array}{l}0.97(0.74- \\
1.27)\end{array}$ & 0.86 \\
\hline & Public Health & $55(85.9)$ & $9(14.1)$ & $\begin{array}{l}1.12(0.99 \\
-1.25)\end{array}$ & 0.1 \\
\hline & Dentistry & $14(82.4)$ & $3(17.6)$ & $\begin{array}{l}1.05(0.84- \\
1.32)\end{array}$ & 0.66 \\
\hline & Other & $22(84.6)$ & $4(15.4)$ & $\begin{array}{l}1.08(0.91- \\
1.29)\end{array}$ & 0.41 \\
\hline Years of practice & Mean (sd) & $11.6(10.8)$ & $9.4(8.6)$ & & 0.04 \\
\hline \multirow[t]{2}{*}{ State where you are practicing } & $\begin{array}{l}\text { Khartoum } \\
\text { State }\end{array}$ & $245(76.3)$ & $76(23.7)$ & \multirow[t]{2}{*}{$\begin{array}{l}0.629(0 \\
.34-1.14)\end{array}$} & \multirow[t]{2}{*}{0.12} \\
\hline & $\begin{array}{l}\text { Other than } \\
\text { Khartoum } \\
\text { State }^{2}\end{array}$ & $82(83.7)$ & $16(16.3)$ & & \\
\hline Any training on COVID-19 & Yes & 167(82.3) & $36(17.7)$ & $\begin{array}{l}1.60(1.00- \\
2.569)\end{array}$ & 0.04 \\
\hline
\end{tabular}




\begin{tabular}{|c|c|c|c|c|c|}
\hline \multirow[t]{2}{*}{ Characteristics } & \multirow[t]{2}{*}{ Value } & \multicolumn{2}{|c|}{$\begin{array}{l}\text { Vaccine } \\
\text { acceptance/refusal ( } \mathrm{N} \\
\left.=421^{1}\right)(\%)\end{array}$} & \multirow[t]{2}{*}{$\begin{array}{l}\text { Unadjusted } \\
\text { OR }(95 \% \\
\mathrm{Cl})\end{array}$} & \multirow[t]{2}{*}{ p } \\
\hline & & Accept (\%) & $\begin{array}{l}\text { Refuse } \\
(\%)\end{array}$ & & \\
\hline & $\mathrm{No}^{2}$ & 162(74.3) & $56(25.7)$ & & \\
\hline \multirow[t]{5}{*}{$\begin{array}{l}\text { Self-rated Knowledge about } \\
\text { COVID-19 }\end{array}$} & Weak & $4(57.1)$ & $3(42.9)$ & $\begin{array}{l}0.67(0.12- \\
3.83)\end{array}$ & 0.65 \\
\hline & Good & $14(66.7)$ & $7(33.3)$ & $\begin{array}{l}0.57(0.12- \\
2.70)\end{array}$ & 0.48 \\
\hline & Moderate & $79(69.9)$ & $34(30.1)$ & $\begin{array}{l}0.26(0.06- \\
1.23)\end{array}$ & 0.09 \\
\hline & Very good & 138(83.6) & $27(16.4)$ & $\begin{array}{l}0.30(0.06- \\
1.43)\end{array}$ & 0.13 \\
\hline & Excellent $^{2}$ & $94(81.7)$ & 21(18.3) & & \\
\hline \multirow[t]{5}{*}{$\begin{array}{l}\text { Self-rated Knowledge about } \\
\text { COVID-19 Vaccination }\end{array}$} & Weak & $38(59.4)$ & $26(40.6)$ & $\begin{array}{l}0.50(0.25- \\
1.00)\end{array}$ & 0.05 \\
\hline & Good & $62(74.7)$ & $21(25.3)$ & $\begin{array}{l}0.34(0.17- \\
0.66)\end{array}$ & 0.00 \\
\hline & Moderate & 103(81.1) & $24(18.9)$ & $\begin{array}{l}0.28(0.14- \\
0.59)\end{array}$ & 0.00 \\
\hline & Very good & 83(83.8) & $16(16.2)$ & $\begin{array}{l}0.17(0.06- \\
0.49)\end{array}$ & 0.00 \\
\hline & Excellent $^{2}$ & 43(89.6) & $5(10.4)$ & & \\
\hline \multirow[t]{2}{*}{ History of COVID-19 infection } & Yes & $67(77.0)$ & $20(23.0)$ & \multirow{2}{*}{$\begin{array}{l}0.92(0 \\
.52-1.61)\end{array}$} & \multirow[t]{2}{*}{0.77} \\
\hline & $\mathrm{No}^{2}$ & $262(78.4)$ & $72(21.6)$ & & \\
\hline \multirow{2}{*}{$\begin{array}{l}\text { Have Any of family members } \\
\text { know been diagnosed with COVID- } \\
\text { 19? }\end{array}$} & Yes & $120(78.4 \%)$ & $33(21.6)$ & \multirow{2}{*}{$\begin{array}{l}1.02(0 \\
.63-1.66)\end{array}$} & \multirow[t]{2}{*}{0.91} \\
\hline & $\mathrm{No}^{2}$ & 209(78.0) & $59(22.0)$ & & \\
\hline \multirow[t]{2}{*}{$\begin{array}{l}\text { Have you directly or indirectly been } \\
\text { taking care of the COVID-19 } \\
\text { patients? }\end{array}$} & Yes & 183(82.8) & $38(17.2)$ & $\begin{array}{l}1.78 \\
(1.11- \\
2.84)\end{array}$ & 0.01 \\
\hline & $\mathrm{No}^{2}$ & $146(73.0)$ & $54(27.0)$ & & \\
\hline \multicolumn{6}{|c|}{1 respondents who answered" May Be" were excluded from the analysis. } \\
\hline
\end{tabular}


Page 11/25 
Table 2

Bivariate analysis for Health Believe Model as Determinants of COVID-19 vaccine acceptance or refusal among the 250 participants who responded yes/no excluding those who responded maybe.

\begin{tabular}{|c|c|c|c|c|c|}
\hline Determinants & Question & $\begin{array}{l}\text { Accept/Yes } \\
(\%)\end{array}$ & $\begin{array}{l}\text { Refuse/No } \\
\text { (\%) }\end{array}$ & $\begin{array}{l}\text { Unadjusted } \\
\text { OR (95\% Cl) }\end{array}$ & $P$ \\
\hline $\begin{array}{l}\text { Perceived } \\
\text { susceptibility }\end{array}$ & $\begin{array}{l}\text { I believe am at risk to get } \\
\text { COVID-19 infection }{ }^{1}\end{array}$ & 307(79.9) & $77(20.1)$ & $\begin{array}{l}2.72(1.35- \\
5.49)\end{array}$ & 0.00 \\
\hline $\begin{array}{l}\text { Perceived } \\
\text { benefit }\end{array}$ & I don't believe vaccine works & $26(51.0)$ & $25(49.0)$ & $\begin{array}{l}0.1(0.05- \\
0.20)\end{array}$ & 0.00 \\
\hline \multirow{2}{*}{$\begin{array}{l}\text { Perceived } \\
\text { harm (Over } \\
\text { All) }\end{array}$} & High & 13(34.2) & $25(65.8)$ & $\begin{array}{l}0.11(0.05- \\
0.23)\end{array}$ & 0.00 \\
\hline & Low & $316(82.5)$ & $67(17.5)$ & & \\
\hline \multirow[t]{2}{*}{$\begin{array}{l}\text { Perceived } \\
\text { barriers }\end{array}$} & $\begin{array}{l}\text { I don't trust pharmaceutical } \\
\text { companies making the vaccine }{ }^{1}\end{array}$ & $37(42.0)$ & $51(58.0)$ & $\begin{array}{l}0.16(0.08- \\
0.31)\end{array}$ & 0.00 \\
\hline & $\begin{array}{l}\text { I don't trust the agents / } \\
\text { government overseeing COVID- } \\
19 \text { vaccination process }{ }^{1}\end{array}$ & $43(48.3)$ & $46(51.7)$ & $\begin{array}{l}0.31(0.17- \\
0.58)\end{array}$ & 0.00 \\
\hline \multirow[t]{4}{*}{$\begin{array}{l}\text { Perceived } \\
\text { severity }\end{array}$} & Mild & 165(79.7) & $42(20.3)$ & $\begin{array}{l}0.51(0.24- \\
1.11)\end{array}$ & 0.09 \\
\hline & Moderate & $69(88.5)$ & $9(11.5)$ & $\begin{array}{l}0.52(0.19- \\
1.39)\end{array}$ & 0.19 \\
\hline & severe & $38(88.4)$ & $5(11.6)$ & $\begin{array}{l}2.48(1.45- \\
4.25)\end{array}$ & 0.00 \\
\hline & Not Concerned ${ }^{3}$ & $57(61.3)$ & $36(38.7)$ & & \\
\hline \multirow[t]{3}{*}{$\begin{array}{l}\text { Cues to } \\
\text { action }\end{array}$} & To prevent COVID-19 in myself & $145(96.7)$ & $5(3.3)$ & $\begin{array}{l}13.39(5.30- \\
33.88)\end{array}$ & 0.00 \\
\hline & $\begin{array}{l}\text { To prevent COVID-19 in friends } \\
\text { and family Members }\end{array}$ & 183(96.8) & $6(3.2)$ & $\begin{array}{l}17.55(7.45- \\
41.32)\end{array}$ & 0.00 \\
\hline & $\begin{array}{l}\text { To prevent COVID-19 in } \\
\text { community }\end{array}$ & 208(95) & $11(5)$ & $\begin{array}{l}12.35(6.32- \\
24.11)\end{array}$ & 0.00 \\
\hline \multicolumn{6}{|c|}{${ }^{1}$ reference $=$ disagree } \\
\hline \multicolumn{6}{|c|}{$\begin{array}{l}\text { 2Perceived harm were described as "high" if the answer was "Agree" to all three items constructs, } \\
\text { otherwise was described as "low" }\end{array}$} \\
\hline
\end{tabular}

Also, Bivariate analysis (Table 3 ) showed a significant and positive association between HCWs vaccine acceptance and their perceived susceptibility to COVID-19 infection (OR $=2.72,95 \% \mathrm{Cl}: 1.35-5.49, P=$ 
$0.00)$, perceived severity of the infection $(\mathrm{OR}=2.48,95 \% \mathrm{Cl}: 1.45-4.25, \mathrm{P}=0.00)$, and the perceived benefit from the vaccine. HCWs acceptance for the vaccine significantly reduced with increased perceived harm from the vaccine ( $\mathrm{OR}=0.11,95 \% \mathrm{Cl}: 0.05-0.23, P=0.00)$, lack of confidence in the vaccine producers (OR $=0.16,95 \% \mathrm{Cl}: 0.08-0.31, P=0.00)$, the organizations and the government supervising COVID-19 vaccination process $(\mathrm{OR}=0.31,95 \% \mathrm{Cl}: 0.17-0.58, P=0.00)($ Table 3$)$. 
Table 3

Bivariate analysis for Health Believe Model as Determinants of COVID-19 vaccine acceptance or refusal among the 250 participants who responded yes/no excluding those who responded maybe.

\begin{tabular}{|c|c|c|c|c|c|}
\hline \multicolumn{2}{|l|}{ Determinants } & \multirow{2}{*}{$\begin{array}{l}\text { Accept } \\
(\%) \\
307(79.9)\end{array}$} & \multirow{2}{*}{$\begin{array}{l}\text { Refuse } \\
(\%) \\
77(20.1)\end{array}$} & \multirow{2}{*}{$\begin{array}{l}\text { Unadjusted } \\
\text { OR (95\% Cl) } \\
2.72(1.35- \\
5.49)\end{array}$} & \multirow{2}{*}{$\begin{array}{l}P \\
0.00\end{array}$} \\
\hline $\begin{array}{l}\text { Perceived } \\
\text { susceptibility }\end{array}$ & $\begin{array}{l}\text { I believe am at risk to get COVID-19 } \\
\text { infection }{ }^{1}\end{array}$ & & & & \\
\hline $\begin{array}{l}\text { Perceived } \\
\text { benefit }\end{array}$ & I don't believe vaccine works & $26(51.0)$ & $25(49.0)$ & $\begin{array}{l}0.1(0.05- \\
0.20)\end{array}$ & 0.00 \\
\hline \multirow{2}{*}{$\begin{array}{l}\text { Perceived } \\
\text { harm (Over } \\
\text { All) }\end{array}$} & High & 13(34.2) & $25(65.8)$ & $\begin{array}{l}0.11(0.05- \\
0.23)\end{array}$ & 0.00 \\
\hline & Low & $316(82.5)$ & $67(17.5)$ & & \\
\hline \multirow[t]{2}{*}{$\begin{array}{l}\text { Perceived } \\
\text { barriers }\end{array}$} & $\begin{array}{l}\text { I don't trust pharmaceutical } \\
\text { companies producing the vaccine }^{1}\end{array}$ & $37(42.0)$ & $51(58.0)$ & $\begin{array}{l}0.16(0.08- \\
0.31)\end{array}$ & 0.00 \\
\hline & $\begin{array}{l}\text { I don't trust the organizations / } \\
\text { government supervising COVID-19 } \\
\text { vaccination process }\end{array}$ & $43(48.3)$ & $46(51.7)$ & $\begin{array}{l}0.31(0.17- \\
0.58)\end{array}$ & 0.00 \\
\hline \multirow[t]{4}{*}{$\begin{array}{l}\text { Perceived } \\
\text { severity }\end{array}$} & Mild & $165(79.7)$ & $42(20.3)$ & $\begin{array}{l}0.51(0.24- \\
1.11)\end{array}$ & 0.09 \\
\hline & Moderate & $69(88.5)$ & $9(11.5)$ & $\begin{array}{l}0.52(0.19- \\
1.39)\end{array}$ & 0.19 \\
\hline & severe & $38(88.4)$ & $5(11.6)$ & $\begin{array}{l}2.48(1.45- \\
4.25)\end{array}$ & 0.00 \\
\hline & Not Concerned $^{3}$ & $57(61.3)$ & $36(38.7)$ & & \\
\hline \multirow[t]{3}{*}{$\begin{array}{l}\text { Cues to } \\
\text { action }\end{array}$} & To prevent COVID-19 in myself & 145(96.7) & $5(3.3)$ & $\begin{array}{l}13.39(5.30- \\
33.88)\end{array}$ & 0.00 \\
\hline & $\begin{array}{l}\text { To prevent COVID-19 in friends and } \\
\text { family Members }\end{array}$ & 183(96.8) & $6(3.2)$ & $\begin{array}{l}17.55(7.45- \\
41.32)\end{array}$ & 0.00 \\
\hline & To prevent COVID-19 in community & 208(95) & $11(5)$ & $\begin{array}{l}12.35(6.32- \\
24.11)\end{array}$ & 0.00 \\
\hline \multicolumn{6}{|c|}{${ }^{1}$ reference $=$ disagree } \\
\hline \multicolumn{6}{|c|}{$\begin{array}{l}\text { 2Perceived harm were described as "high" if the answer was "Agree" to all three items constructs, } \\
\text { otherwise was described as "low" }\end{array}$} \\
\hline
\end{tabular}


Table 3

Bivariate analysis for Socio-demographic, profession, and health characteristics as determinants of COVID-19 vaccine acceptance and/or refusal.

\begin{tabular}{|c|c|c|c|c|c|}
\hline \multirow[t]{2}{*}{ Characteristics } & \multirow[t]{2}{*}{ Value } & \multicolumn{2}{|c|}{$\begin{array}{l}\text { Vaccine } \\
\text { acceptance/refusal ( } \mathrm{N} \\
\left.=421^{1}\right)(\%)\end{array}$} & \multirow[t]{2}{*}{$\begin{array}{l}\text { Unadjusted } \\
\text { OR ( } 95 \% \\
\mathrm{Cl})\end{array}$} & \multirow[t]{2}{*}{$\mathbf{p}$} \\
\hline & & Accept (\%) & $\begin{array}{l}\text { Refuse } \\
\text { (\%) }\end{array}$ & & \\
\hline Age & Mean (sd) & $36.5(11.5)$ & $34.1(9.6)$ & & 0.04 \\
\hline \multirow[t]{2}{*}{ Gender } & Male & $181(85.8)$ & $30(14.2)$ & \multirow{2}{*}{$\begin{array}{l}2.52(1.55- \\
4.11)\end{array}$} & \multirow[t]{2}{*}{0.00} \\
\hline & Female $^{2}$ & 148(70.5) & $62(29.5)$ & & \\
\hline \multirow[t]{2}{*}{ Medical Co-morbidities } & Yes & $93(79.5)$ & $24(20.5)$ & \multirow{2}{*}{$\begin{array}{l}1.11 \\
(0.66- \\
1.88)\end{array}$} & \multirow[t]{2}{*}{0.68} \\
\hline & $\mathrm{No}^{2}$ & $236(77.6)$ & $68(22.4)$ & & \\
\hline \multirow{2}{*}{$\begin{array}{l}\text { Taking Immune suppressing } \\
\text { medications }\end{array}$} & Yes & $9(69.2)$ & $4(30.8)$ & \multirow{2}{*}{$\begin{array}{l}0.61(0 \\
.18-2.06)\end{array}$} & \multirow[t]{2}{*}{0.49} \\
\hline & $\mathrm{No}^{2}$ & $316(78.4)$ & $87(21.6)$ & & \\
\hline \multirow[t]{7}{*}{ Health Profession } & Medicine & 190(79.2) & $50(20.8)$ & $\begin{array}{l}1.03(0.93- \\
1.14)\end{array}$ & 0.5 \\
\hline & Nursing & $4(28.6)$ & 10(71.4) & $\begin{array}{l}0.35(0.15- \\
0.82)\end{array}$ & 0.00 \\
\hline & Pharmacy & $31(72.1)$ & 12(27.9) & $\begin{array}{l}0.91(0.75- \\
1.10)\end{array}$ & 0.31 \\
\hline & $\begin{array}{l}\text { Medical } \\
\text { laboratory }\end{array}$ & $13(76.5)$ & $4(23.5)$ & $\begin{array}{l}0.97(0.74- \\
1.27)\end{array}$ & 0.86 \\
\hline & Public Health & $55(85.9)$ & $9(14.1)$ & $\begin{array}{l}1.12(0.99 \\
-1.25)\end{array}$ & 0.1 \\
\hline & Dentistry & $14(82.4)$ & $3(17.6)$ & $\begin{array}{l}1.05(0.84- \\
1.32)\end{array}$ & 0.66 \\
\hline & Other & $22(84.6)$ & $4(15.4)$ & $\begin{array}{l}1.08(0.91- \\
1.29)\end{array}$ & 0.41 \\
\hline Years of practice & Mean (sd) & $11.6(10.8)$ & $9.4(8.6)$ & & 0.04 \\
\hline \multirow[t]{2}{*}{ State where you are practicing } & $\begin{array}{l}\text { Khartoum } \\
\text { State }\end{array}$ & $245(76.3)$ & $76(23.7)$ & \multirow[t]{2}{*}{$\begin{array}{l}0.629(0 \\
.34-1.14)\end{array}$} & \multirow[t]{2}{*}{0.12} \\
\hline & $\begin{array}{l}\text { Other than } \\
\text { Khartoum } \\
\text { State }^{2}\end{array}$ & $82(83.7)$ & $16(16.3)$ & & \\
\hline Any training on COVID-19 & Yes & 167(82.3) & $36(17.7)$ & $\begin{array}{l}1.60(1.00- \\
2.569)\end{array}$ & 0.04 \\
\hline
\end{tabular}




\begin{tabular}{|c|c|c|c|c|c|}
\hline \multirow[t]{2}{*}{ Characteristics } & \multirow[t]{2}{*}{ Value } & \multicolumn{2}{|c|}{$\begin{array}{l}\text { Vaccine } \\
\text { acceptance/refusal ( } \mathrm{N} \\
\left.=421^{1}\right)(\%)\end{array}$} & \multirow[t]{2}{*}{$\begin{array}{l}\text { Unadjusted } \\
\text { OR }(95 \% \\
\mathrm{Cl})\end{array}$} & \multirow[t]{2}{*}{$\mathrm{p}$} \\
\hline & & Accept (\%) & $\begin{array}{l}\text { Refuse } \\
\text { (\%) }\end{array}$ & & \\
\hline & $\mathrm{No}^{2}$ & 162(74.3) & $56(25.7)$ & & \\
\hline \multirow[t]{5}{*}{$\begin{array}{l}\text { Self-rated Knowledge about } \\
\text { COVID-19 }\end{array}$} & Weak & $4(57.1)$ & $3(42.9)$ & $\begin{array}{l}0.67(0.12- \\
3.83)\end{array}$ & 0.65 \\
\hline & Good & $14(66.7)$ & 7(33.3) & $\begin{array}{l}0.57(0.12- \\
2.70)\end{array}$ & 0.48 \\
\hline & Moderate & $79(69.9)$ & $34(30.1)$ & $\begin{array}{l}0.26(0.06- \\
1.23)\end{array}$ & 0.09 \\
\hline & Very good & 138(83.6) & $27(16.4)$ & $\begin{array}{l}0.30(0.06- \\
1.43)\end{array}$ & 0.13 \\
\hline & Excellent $^{2}$ & $94(81.7)$ & $21(18.3)$ & & \\
\hline \multirow[t]{5}{*}{$\begin{array}{l}\text { Self-rated Knowledge about } \\
\text { COVID-19 Vaccination }\end{array}$} & Weak & $38(59.4)$ & $26(40.6)$ & $\begin{array}{l}0.50(0.25- \\
1.00)\end{array}$ & 0.05 \\
\hline & Good & $62(74.7)$ & $21(25.3)$ & $\begin{array}{l}0.34(0.17- \\
0.66)\end{array}$ & 0.00 \\
\hline & Moderate & 103(81.1) & $24(18.9)$ & $\begin{array}{l}0.28(0.14- \\
0.59)\end{array}$ & 0.00 \\
\hline & Very good & 83(83.8) & $16(16.2)$ & $\begin{array}{l}0.17(0.06- \\
0.49)\end{array}$ & 0.00 \\
\hline & Excellent $^{2}$ & 43(89.6) & $5(10.4)$ & & \\
\hline \multirow[t]{2}{*}{ History of COVID-19 infection } & Yes & $67(77.0)$ & $20(23.0)$ & \multirow{2}{*}{$\begin{array}{l}0.92(0 \\
.52-1.61)\end{array}$} & \multirow[t]{2}{*}{0.77} \\
\hline & $\mathrm{No}^{2}$ & $262(78.4)$ & $72(21.6)$ & & \\
\hline \multirow{2}{*}{$\begin{array}{l}\text { Have Any of family members } \\
\text { know been diagnosed with COVID- } \\
19 \text { ? }\end{array}$} & Yes & $120(78.4 \%)$ & $33(21.6)$ & \multirow{2}{*}{$\begin{array}{l}1.02(0 \\
.63-1.66)\end{array}$} & \multirow[t]{2}{*}{0.91} \\
\hline & $\mathrm{No}^{2}$ & $209(78.0)$ & $59(22.0)$ & & \\
\hline \multirow[t]{2}{*}{$\begin{array}{l}\text { Have you directly or indirectly been } \\
\text { taking care of the COVID- } 19 \\
\text { patients? }\end{array}$} & Yes & 183(82.8) & $38(17.2)$ & $\begin{array}{l}1.78 \\
(1.11- \\
2.84)\end{array}$ & 0.01 \\
\hline & $\mathrm{No}^{2}$ & $146(73.0)$ & $54(27.0)$ & & \\
\hline
\end{tabular}




\section{Determinant of COVID-19 vaccine acceptability among HCWs}

Following bivariate analysis, the sociodemographic characteristics, professional characteristics and health belief model predictors were fitted with multiple logistic regression (Table 4). Only three sociodemographic and professional characteristics continued to be significant predictors for HCWs' vaccine acceptance namely: gender, the nursing profession, and self-rated knowledge about the vaccine. Males were twice more likely to take the vaccine compared to females $(\mathrm{OR}=2.4,95 \% \mathrm{Cl}: 1.23-4,63), \mathrm{p}<$ $0.01)$. Nursing profession continued to be negative predictor for acceptance $(\mathrm{OR}=0.04,95 \% \mathrm{Cl}: 0.01-0.24$, $P=0.00$ ). In addition, self-rated knowledge about COVID-19 vaccination continued to be significantly and negatively associated with HCWs acceptance of the vaccine. Having a good ( $\mathrm{OR}=0.45,95 \% \mathrm{Cl}$ : $0.08-$ $2.46, \mathrm{P}=0.01$ ), a moderate $\mathrm{OR}=0.56,95 \% \mathrm{Cl}: 0.27-1.16, \mathrm{P}=0.02$ ), and a very good $\mathrm{OR}=0.36$, $95 \% \mathrm{Cl}: 0.19-0.81, \mathrm{P}=0.02$ ). 
Table 4

Multivariable logistic regression for Determinants of COVID-19 vaccine acceptance

\begin{tabular}{|c|c|c|c|}
\hline \multicolumn{2}{|c|}{ Determinants of vaccine acceptance (Accept /Refuse) } & $\begin{array}{l}\text { aOR }(95 \% \mathrm{Cl} \\
\text { aOR) }\end{array}$ & $p$ \\
\hline \multicolumn{2}{|l|}{ Age (M) (SD) } & $\begin{array}{l}1.01(0.98- \\
1.05)\end{array}$ & 0.32 \\
\hline \multicolumn{2}{|l|}{ Years of practice (M) (SD) } & $\begin{array}{l}0.99(0.92- \\
1.06)\end{array}$ & 0.66 \\
\hline \multirow[t]{2}{*}{ Gender } & Male & \multirow{2}{*}{$\begin{array}{l}2.39(1.23- \\
4.63)\end{array}$} & \multirow[t]{2}{*}{0.01} \\
\hline & Female $^{1}$ & & \\
\hline \multirow[t]{7}{*}{ Health Profession } & Medicine & $\begin{array}{l}0.25(0.08- \\
0.74)\end{array}$ & 0.01 \\
\hline & Nursing & $\begin{array}{l}0.04(0.01- \\
0.24)\end{array}$ & 0.00 \\
\hline & Pharmacy & $\begin{array}{l}0.22(0.05- \\
0.83)\end{array}$ & 0.03 \\
\hline & Medical laboratory & $\begin{array}{l}0.78(0.12- \\
4.94)\end{array}$ & 0.80 \\
\hline & Public Health & $\begin{array}{l}0.90(.25- \\
3.22)\end{array}$ & 0.10 \\
\hline & Dentistry & $\begin{array}{l}0.41(0.06- \\
2.80)\end{array}$ & 0.37 \\
\hline & others & $\begin{array}{l}0.45(0.08- \\
2.46)\end{array}$ & 0.36 \\
\hline \multirow[t]{2}{*}{ Any training on COVID-19 } & Yes & \multirow{2}{*}{$\begin{array}{l}1.32(0.58- \\
3.00)\end{array}$} & \multirow[t]{2}{*}{0.51} \\
\hline & $\mathrm{No}^{1}$ & & \\
\hline \multirow[t]{5}{*}{$\begin{array}{l}\text { Self- rated Knowledge about } \\
\text { COVID-19 }\end{array}$} & Weak & $\begin{array}{l}1.02(0.28- \\
3.65)\end{array}$ & 0.98 \\
\hline & Good & $\begin{array}{l}0.91(0.27- \\
3.03)\end{array}$ & 0.87 \\
\hline & Moderate & $\begin{array}{l}0.68(0.17- \\
2.69)\end{array}$ & 0.58 \\
\hline & Very good & $\begin{array}{l}0.63(0.09- \\
4.03)\end{array}$ & 0.62 \\
\hline & Excellent $^{1}$ & & \\
\hline $\begin{array}{l}\text { Self- rated Knowledge about } \\
\text { COVID-19 Vaccination }\end{array}$ & Weak & $\begin{array}{l}0.41(0.06- \\
2.80)\end{array}$ & 0.12 \\
\hline
\end{tabular}


Good

Moderate

Very good

Excellent ${ }^{1}$

Directly or indirectly been

taking care of the COVID-19 patients

Perceived susceptibility

Yes

$\mathrm{No}^{1}$

yes
$1.03(0.47-$ 2.35)

$0.45(0.0$
$2.46)$

$0.56(0.27-$ 1.16)

$0.36(0.19-$ $0.81)$
0.01

0.02

0.02

$1.31(0.47-$

3.63)

$\mathrm{No}^{1}$

Perceived Benefit

$1.51(0.55-$

4.14)

perceived barriers

I don't trust pharmaceutical companies

making the vaccine ${ }^{2}$

$0.19(0.07-$

$0.47)$

I don't trust the organizations / government

$0.59(0.25-$ 1.41)

Perceived harm

I don't believe the vaccine is safe

$0.35(0.13-$

$0.96)$

Perceived Severity

Mild

$0.33(0.13-$

$0.86)$

Moderate

$0.50(0.14-$

1.82)

high

$1.65(0.76-$

3.61)

Cues to action (taking the vaccine)

To prevent COVID-19 in myself 3

$52.50(15.90-\quad 0.00$ 172.60)

To prevent COVID-19 in friends and family Members 3

$85.09(27.30-\quad 0.00$ 264.70)

To prevent COVID-19 in community 3

$54.55(20.89-\quad 0.00$ 142.43)

${ }^{1}$ Reference category

2 Reference category = Disagree 
Among the Health Beliefs determinants, only perceived harm, perceived lack of confidence in vaccine producers, and all cues to take the vaccine continued to be statistically significant. Acceptability was significantly reduced with lack of confidence in the vaccine producers, $\mathrm{OR}=0.19,95 \% \mathrm{Cl}: 0.07-0.47, P=$ 0.00) (Table 4). This was even higher than the significant negative impact of perceived harms from the vaccine (OR $=0.35,95 \% \mathrm{Cl}: 0.13-0.96, P=0.04)$. The main cue to accepting the vaccine by HCWs was to protect their families $(\mathrm{OR}=85.09,95 \% \mathrm{Cl}$ : $27.30-264.70, \mathrm{P}=0.00)($ Table 4$)$. A breakdown of the analysis for each variable can be found in Table 4.

\section{Hows' Concerns About Covid-19 Vaccination In Sudan}

Besides the participants concerns about the safety and the possible side effects of the vaccine, some additional concerns about other aspects were expressed in the comments section of the questionnaire as follows:

A 37 years old male doctor. Stated "I think there is a hidden agenda behind COVID-19 vaccine", and a 35 years old female doctor was concerned about the effect of vaccine storing conditions on its safety and efficacy "I am concerned about the storage of the vaccine". Furthermore, two participants were worried about potential corruption related to vaccine distribution, which may lead to unequal distribution of the vaccine. Moreover, many participants emphasized the importance of information dissemination and increasing the awareness of the population to fight false rumors about the vaccine. Additionally, a female doctor suggested that all HCWs be tested for the COVID-19 IgG before vaccination. Lastly, a 39 years old female doctor stated that "For me, to be vaccinated or not is mainly dependent on the type of vaccine available. I do prefer the Oxford vaccine rather than the Pitfizer vaccine".

\section{Discussion}

The healthcare workers were among the most affected groups by the ongoing COVID-19 pandemic worldwide (20). Since its start in Sudan in March 2020, HCWs were burdened with COVID-19 mortality and morbidity $(3,4,21)$. The current surge of COVID-19 cases leads to severe disruption of collateral health services $(5,22)$. Implementing preventive measures to protect $\mathrm{HCW}$ was a real challenge, which was further complicated by the global shortage of Personal Protective Equipments (PPEs) (23). The rapid development of the COVID-19 vaccine has raised as one of the major strategies to reduce morbidity and mortality due to this pandemic $(6,24)$. Low- and middle-income countries (LMICs) including Sudan were challenged to strategically benefit from the vaccine to stop or at least to slow the current pandemic due to difficulties in achieving the optimal coverage among the most at-risk populations, mainly the front-line responders (25). Globally, efforts have been made for equitable access to the vaccine, with a main global understanding that "no one is safe until everyone is safe'. Sudan has joined the COVAX AMC initiative in December 2020 and received 828,000 doses of the AstraZeneca vaccine through the initiative support (18), which is only enough to vaccinate less than $1 \%$ of the country population. Considering this limitation of vaccine supply, the national committee for COVID-19 deployment and vaccination plan has 
prioritized the HCWs and most vulnerable groups (elders with co-morbidities) for the first phase of the vaccination to maintain the health service, reducing deaths and disease severity among the vulnerable group. Despite these efforts to make vaccines available for this key target group, vaccine acceptance by the HCWs would significantly affect its uptake. COVID-19 vaccination hesitancy has been of increasing concern in many countries $(13,14)$. In this survey, we measured the extent of acceptability of COVID-19 vaccination among HCWs in Sudan. We also identified key factors that could affect HCW's decision to take the vaccine. This is expected to support the development of evidence-based COVID-19 vaccination advocacy to ensure optimal acceptance of COVID-19 vaccine among HCWs in Sudan

The majority of respondents were young with average age of 35.3 years, direct or indirect caretakers of COVID-19's patients and $20 \%$ of them had a history of COVID-19 infection. Females represented more than half of the respondents and this matches the gender representation of the health workforce in Sudan. Sixteen percent expressed absolute refusal, which is double the percentage reported by HCWs in the US $(14,26)$. In contrast, $57 \%$ of the Sudanese HCWs will take the vaccine as soon as it is available. This relatively high acceptance rate is comparable to what has been reported by UNICEF in Ghana (27), and double the acceptance rate reported among Congolese HCWs (13). The latter has the lowest reported HCWs' vaccine acceptance globally (11). Nurses' likelihood to accept the vaccine was almost zero. They were the category of HCWs with the least acceptance rate for the vaccine.

To increase COVID-19 vaccine acceptance, positive reinforcement of public perceptions through effective communications of the correct and up-to-date evidence on the safety and effectiveness of the vaccines is crucial. Exposure to misinformation about the vaccine can lower COVID-19 acceptance by up to 6\% (28). In this study, HCWs' acceptance was inversely correlated with their self-rated Knowledge about COVID-19 vaccination. It was clear that HCWs have built negative perceptions regarding the COVID-19 vaccine. This was translated into concerns regarding the process of development and safety of the vaccine, as more than half of the participants were worried about the vaccine's side effects. More than a quarter were worried about how rapidly the vaccine was developed. Perceived mistrust towards vaccine manufacturers and increased perceived harms from the vaccine reduced the vaccine acceptance by almost $80 \%$ and $65 \%$, respectively. However, this is a global challenge given the fact that the vaccine for COVID-19 was developed within the shortest time in human history. This calls for putting extra effort to build public trust for the vaccine. The advocacy phase should focus on providing up-to-date information about vaccine safety. Implementation-wise, well-functioning adverse events and rumors tracking systems are vital.

Female HCWs were twice less likely to take the vaccine compared to their male colleagues. This might be explained by the gender disparities in perceived vaccine safety. Adverse events from COVID-19 vaccines have been unduly reported among Women compared to men, and it has been excessively covered in the news (29-31). The latter could have disproportionately built a negative perception of vaccine safety among females compared to males. It is crucial to take gender differences into account when addressing COVID-19 vaccine acceptance, as women represent the majority of the healthcare workforce in the country (26). This high hesitancy puts them at a higher risk of COVID-19 morbidity and mortality. 
In conclusion, the acceptability of COVID-19 vaccination among HCWs in Sudan was average and comparable to others globally. Vaccine advocacy among HCWs needs to be aligned with the identified determinants. Acceptability was negatively determined by the female gender, the nursing profession, and knowledge about COVID-19 vaccination. Also, acceptability was significantly reduced by the increased perceived harm from the vaccine and the increased mistrust for the vaccine manufacture.

Strengths and Limitations: Little is known about the acceptability of COVID-19 vaccination among HCWs in LIMICs. The major strength of this study is that this survey is the first post vaccine availability survey to address determinants of COVID-19 vaccination acceptability among HCWs in Sudan. A potential limitation of this study is that the questionnaire was distributed in an online format which could introduce selection bias by excluding HCWs who do not have access to the internet.

\section{Declarations}

Conflict of interest: Authors have no conflict of interest to declare

Funding: This research did not receive any specific grant from funding agencies in the public, commercial, or not-for-profit sector.

\section{Acknowledgments:}

We acknowledge health care societies, colleagues, and academics for participating in the distribution of the questionnaire.

\section{References}

1. The World Health Organization (WHO). Novel Coronavirus (2019-nCoV) situation reports [Internet]. 2020 [cited 2020 Sep 5]. Available from: https://www.who.int/emergencies/diseases/novel-coronavirus2019/situation-reports

2. The World Health Organization (WHO). WHO Coronavirus (COVID-19) Dashboard [Internet]. 2021 [cited 2021 Jun 27]. Available from: https://covid19.who.int

3. Bandyopadhyay S, Baticulon RE, Kadhum M, Alser M, Ojuka DK, Badereddin Y, et al. Infection and mortality of healthcare workers worldwide from COVID-19: a systematic review. BMJ Global Health. 2020 Dec 1;5(12):e003097.

4. Aljak ER, Eldigail M, Mahmoud I, Elhassan RM, Elduma A, Ibrahim AA, et al. The first laboratoryconfirmed imported infections of SARS-CoV-2 in Sudan. Transactions of The Royal Society of Tropical Medicine and Hygiene [Internet]. 2020 Dec 15 [cited 2020 Dec 28];(traa151). Available from: https://doi.org/10.1093/trstmh/traa151 
5. Ahmed A, Mohamed NS, EL-Sadig SM, Fahal LA, Abelrahim ZB, Ahmed ES, et al. COVID-19 in Sudan. The Journal of Infection in Developing Countries. 2021 Mar 7;15(02):204-8.

6. The World Health Organization (WHO). WHO issues its first emergency use validation for a COVID19 vaccine and emphasizes need for equitable global access [Internet]. 2020 [cited 2021 Jun 27]. Available from: https://www.who.int/news/item/31-12-2020-who-issues-its-first-emergency-usevalidation-for-a-covid-19-vaccine-and-emphasizes-need-for-equitable-global-access

7. The World Health Organization (WHO). COVID-19 vaccines [Internet]. [cited 2021 Jun 27]. Available from: https://www.who.int/emergencies/diseases/novel-coronavirus-2019/covid-19-vaccines

8. Dror AA, Eisenbach N, Taiber S, Morozov NG, Mizrachi M, Zigron A, et al. Vaccine hesitancy: the next challenge in the fight against COVID-19. Eur J Epidemiol. 2020 Aug 1;35(8):775-9.

9. The European Centre for Disease Prevention and Control (ECDC). Vaccine hesitancy [Internet]. European Centre for Disease Prevention and Control. 2017 [cited 2021 Jun 27]. Available from: https://www.ecdc.europa.eu/en/immunisation-vaccines/vaccine-hesitancy

10. Karafillakis E, Dinca I, Apfel F, Cecconi S, Würz A, Takacs J, et al. Vaccine hesitancy among healthcare workers in Europe: A qualitative study. Vaccine. 2016 Sep 22;34(41):5013-20.

11. Sallam M. COVID-19 Vaccine Hesitancy Worldwide: A Concise Systematic Review of Vaccine Acceptance Rates. Vaccines. 2021 Feb;9(2):160.

12. Qattan AMN, Alshareef N, Alsharqi O, Al Rahahleh N, Chirwa GC, Al-Hanawi MK. Acceptability of a COVID-19 Vaccine Among Healthcare Workers in the Kingdom of Saudi Arabia. Front Med [Internet]. 2021 [cited 2021 Jun 27];8. Available from:

https://www.frontiersin.org/articles/10.3389/fmed.2021.644300/full

13. Kabamba Nzaji M, Kabamba Ngombe L, Ngoie Mwamba G, Banza Ndala DB, Mbidi Miema J, Luhata Lungoyo C, et al. Acceptability of Vaccination Against COVID-19 Among Healthcare Workers in the Democratic Republic of the Congo. Pragmat Obs Res. 2020 Oct 29;11:103-9.

14. Shekhar R, Sheikh AB, Upadhyay S, Singh M, Kottewar S, Mir H, et al. COVID-19 Vaccine Acceptance among Health Care Workers in the United States. Vaccines. 2021 Feb;9(2):119.

15. Papagiannis D, Rachiotis G, Malli F, Papathanasiou IV, Kotsiou O, Fradelos EC, et al. Acceptability of COVID-19 Vaccination among Greek Health Professionals. Vaccines. 2021 Mar;9(3):200.

16. Gagneux-Brunon A, Detoc M, Bruel S, Tardy B, Rozaire O, Frappe P, et al. Intention to get vaccinations against COVID-19 in French healthcare workers during the first pandemic wave: a crosssectional survey. Journal of Hospital Infection. 2021 Feb 1;108:168-73. 
17. Fu C, Wei Z, Pei S, Li S, Sun X, Liu P. Acceptance and preference for COVID-19 vaccination in health-care workers (HCWs). medRxiv. 2020 Apr 14;2020.04.09.20060103.

18. The United Nations Children's Emergency Fund (UNICEF). Sudan receives first delivery of COVID-19 vaccines with over 800,000 doses [Internet]. 2021 [cited 2021 Jun 27]. Available from:

https://www.unicef.org/press-releases/sudan-receives-first-delivery-covid-19-vaccines-over-800000-doses

19. Wong MCS, Wong ELY, Huang J, Cheung AWL, Law K, Chong MKC, et al. Acceptance of the COVID19 vaccine based on the health belief model: A population-based survey in Hong Kong. Vaccine. 2021 Feb 12;39(7):1148-56.

20. Nguyen LH, Drew DA, Graham MS, Joshi AD, Guo C-G, Ma W, et al. Risk of COVID-19 among frontline health-care workers and the general community: a prospective cohort study. The Lancet Public Health. 2020 Sep 1;5(9):e475-83.

21. Salih MAM, Swar MO. Sudanese doctors continue to offer their lives around the globe fighting coronavirus (COVID-19) pandemic. Sudan J Paediatr. 2020;20(2):96-8.

22. The United Nations Office for the Coordination of Humanitarian Affairs (OCHA). Impact of COVID19 on continuity of health services in Sudan [Internet]. 2020 [cited 2021 Jun 27]. Available from: https://reports.unocha.org/en/country/sudan/card/49cURoYa8t/

23. The World Health Organization (WHO). Shortage of personal protective equipment endangering health workers worldwide [Internet]. 2020 [cited 2021 Jun 27]. Available from:

https://www.who.int/news/item/03-03-2020-shortage-of-personal-protective-equipment-endangeringhealth-workers-worldwide

24. The European Medicines Agency (EMA). EMA recommends first COVID-19 vaccine for authorization in the EU [Internet]. European Medicines Agency. 2020 [cited 2021 Jun 27]. Available from: https://www.ema.europa.eu/en/news/ema-recommends-first-covid-19-vaccine-authorisation-eu

25. El-Sadig SM, Fahal LA, Abdelrahim ZB, Ahmed ES, Mohamed NS, Siddig EE. Impact of COVID-19 on doctors and healthcare providers during the pandemic in Sudan. Transactions of The Royal Society of Tropical Medicine and Hygiene [Internet]. 2021 Feb 6 [cited 2021 May 6];(trab016). Available from: https://doi.org/10.1093/trstmh/trab016

26. The Japan International Cooperation Agency (JICA). The Republic of Sudan: country gender profile. - [Internet]. 2012 [cited 2021 Jun 27]. Available from:

https://openjicareport.jica.go.jp/214/214/214_415_12121810.html

27. The United Nations Children's Emergency Fund (UNICEF). COVID-19 Vaccination Hesitancy in Guyana: Survey Report [Internet]. 2021 [cited 2021 Jun 27]. Available from: 
https://www.unicef.org/guyanasuriname/reports/covid-19-vaccination-hesitancy-survey-report-january2020

28. Loomba S, de Figueiredo A, Piatek SJ, de Graaf K, Larson HJ. Measuring the impact of COVID-19 vaccine misinformation on vaccination intent in the UK and USA. Nat Hum Behav. 2021 Mar;5(3):33748.

29. Menni C, Klaser K, May A, Polidori L, Capdevila J, Louca P, et al. Vaccine side-effects and SARSCoV-2 infection after vaccination in users of the COVID Symptom Study app in the UK: a prospective observational study. The Lancet Infectious Diseases. 2021 Jul 1;21(7):939-49.

30. The European Medicines Agency (EMA). AstraZeneca's COVID-19 vaccine: EMA finds possible link to very rare cases of unusual blood clots with low platelets [Internet]. European Medicines Agency. 2021 [cited 2021 Jun 27]. Available from: https://www.ema.europa.eu/en/news/astrazenecas-covid-19vaccine-ema-finds-possible-link-very-rare-cases-unusual-blood-clots-low-blood

31. The British Broadcasting Corporation (BBC). Covid-19: Netherlands suspends use of AstraZeneca vaccine. BBC News [Internet]. 2021 Mar 15 [cited 2021 Jun 27]; Available from: https://www.bbc.com/news/world-europe-56397157

\section{Supplementary Files}

This is a list of supplementary files associated with this preprint. Click to download.

- Tablesupplementary.docx 